\title{
Identificação de risco de dano no pprocedimento de punção venosa periférica realizado por enfermeiros: Protocolo de Revisão de Escopo
}

\author{
Identification of Risk of Damage in the Procedure of Peripheral Venipuncture Performed by \\ Nurses: A Scope Review Protocol \\ Identificación del riesgo de daño en el procedimiento de punción venosa periférica realizado por \\ enfermeras: Scoping Review Protocol
}

Recebido: 14/06/2021 | Revisado: 19/06/2021 | Aceito: 24/06/2021 | Publicado: 10/07/2021

\author{
Karinne Cristinne da Silva Cunha \\ ORCID: https://orcid.org/0000-0003-4971-9801 \\ Universidade Federal Fluminense, Brasil \\ E-mail: karinnecunha@id.uff.br \\ Elisa Oliveira de Almeida \\ ORCID: https://orcid.org/0000-0002-4617-7165 \\ Universidade Federal Fluminense, Brasil \\ E-mail: elisaalmeida@id.uff.br \\ Érica Brandão de Moraes \\ ORCID: https://orcid.org/0000-0003-3052-158X \\ Universidade Federal Fluminense, Brasil \\ E-mail: ericabrandao@id.uff.br \\ Amanda Cristina Oliveira da Silva \\ ORCID: https://orcid.org/0000-0002-1312-7567 \\ Universidade Federal Fluminense, Brasil \\ E-mail: amandacristinasilva@id.uff.br \\ Danielle Galdino de Paula \\ ORCID: https://orcid.org/0000-0002-0103-6828 \\ Universidade Federal do Estado do Rio de Janeiro, Brasil \\ E-mail: danielle.paula@unirio.br
}

\begin{abstract}
Resumo
Trata-se de um protocolo de revisão de escopo sobre procedimentos invasivos realizados por enfermeiros, e tem como objetivo formalizar o estudo primário. O protocolo define a metodologia que será adotada, sendo esta fundamentada em orientações do Joanna Briggs Institute através das seguintes fases: Elaboração da questão de pesquisa; Construção do protocolo da revisão; Busca das publicações nas bases de dados eletrônicas; Seleção dos artigos; Extração dos dados dos artigos e Síntese e Análise dos dados. Com a revisão, espera-se promover a segurança do paciente e identificar os riscos e eventos adversos nas punções venosas periféricas, realizadas por enfermeiros.

Palavras-chave: Protocolo; Revisão de escopo; Segurança do paciente; Cuidados de enfermagem; Punção venosa periférica; Riscos; Danos.
\end{abstract}

\begin{abstract}
It is a scope review protocol about invasive procedures performed by nurses, and aims to formalize the primary study. The protocol defines the methodology that will be adopted, which is based on guidelines from the Joanna Briggs Institute through the following phases: Elaboration the research question; Construction of the review protocol; Search of publications in electronic databases; Selection of articles; Extraction of data from articles and Synthesis and Analysis of data. With the review, it is expected to promote patient safety and identify risks and adverse events in peripheral venous punctures, performed by nurses.
\end{abstract}

Keywords: Protocol; Scope review; Patient safety; Nursing care; Peripheral venipuncture; Scratchs; Damage.

\section{Resumen}

Se trata de un protocolo de revisión acerca del alcance de los procedimientos invasivos realizados por enfermeras, y tiene como objetivo formalizar el estudio primario. El protocolo define la metodología que se adoptará, la cual se basa en las directrices del Instituto Joanna Briggs a través de las siguientes fases: Elaboración de la pregunta de investigación; Construcción del protocolo de revisión; Búsqueda de publicaciones en bases de datos electrónicas; Selección de artículos; Extracción de datos de artículos y Síntesis y Análisis de datos. Con la revisión, se espera promover la seguridad del paciente e identificar riesgos y eventos adversos en las punciones venosas periféricas, realizadas por enfermeras.

Palabras clave: Protocolo; Revisión del alcance; Seguridad del paciente; Cuidado de enfermera; Venopunción periférica; Arañazos; Daño. 


\section{Introdução}

As abordagens envolvendo o tema sobre segurança do paciente são cada vez mais atuais por conta da sua importância para a qualidade da assistência à saúde em toda a sua complexidade. Entretanto, é uma preocupação que não é atual. Em sua obra Notes on Hospitals, Florence Nightingale já demonstrava uma preocupação para a segurança na assistência prestada: "Pode parecer um princípio estranho anunciar como requisito básico, em um hospital, que não se deve causar dano ao paciente".

A temática segurança do paciente chamou a atenção do mundo e ganhou o status de grave problema de saúde pública, tornando-se prioridade para as instituições de saúde apenas após o conhecimento sobre danos sofridos por pacientes advindos da assistência prestada, o que ocorreu com a publicação do livro To Err Is Human: Building a Safer Health System pelo Institute of Medicine, em 1999, cujo conteúdo expõe uma estratégia abrangente para que pudesse reduzir esses erros, tido como evitáveis (Kohn, Corrigan e Donaldson, 2000) (Sousa, 2014).

No Brasil, somente em 2013, foi instituído o Programa Nacional de Segurança do Paciente (PNSP), por meio da Portaria no 529/2013. Além do PNSP, a RDC no 36 foi publicada no mesmo ano, tornando obrigatória a constituição do Núcleo de Segurança do Paciente (NSP) em serviços públicos e privados (Ministério da Saúde [MS], 2013, b). O Ministério da Saúde instituiu ainda as seis metas básicas de segurança do paciente: identificar corretamente o paciente; melhorar a comunicação entre profissionais de saúde; melhorar a segurança na prescrição, no uso e na administração de medicamentos; assegurar a cirurgia em local de intervenção, procedimento e paciente corretos; e reduzir o risco de quedas e úlcera por pressão (MS, 2014).

Segurança do paciente é um termo definido mundialmente como um conjunto de ações para redução, a um mínimo aceitável, do risco de dano desnecessário associado ao cuidado de saúde. Entende-se que todo cuidado prestado em saúde é passível da ocorrência de incidentes (erros e falhas), que ameaçam a segurança do paciente. Incidentes relacionados ao cuidado de saúde são eventos ou circunstâncias que poderiam ter resultado, ou resultaram, em dano desnecessário ao paciente. Os incidentes classificam-se como: near miss (incidente que não atingiu o paciente; nesse caso o profissional percebe o erro antes de prestar o cuidado ao paciente); incidente sem dano (incidente que atingiu o paciente, mas não causou ano discernível) e incidente com dano, também denominado de evento adversos (ocorre o incidente, e há dano ao paciente). $\mathrm{O}$ dano pode ser leve, moderado, grave e catastrófico, quando leva o indivíduo ao óbito (MS,2013a) (MS, 2014).

A Organização Mundial de Saúde (OMS) em estudo afirmou que dezenas de milhares de pessoas durante o ano, em diversos países, foram afetadas por erros associados à assistência à saúde, hoje conhecido por eventos adversos (EAs). Em países desenvolvidos estima-se que pelo menos $5 \%$ dos pacientes admitidos em hospitais adquirem uma infecção. Os cuidados de saúde inseguros resultam em expressiva morbidade e mortalidade evitáveis, gastos adicionais com a manutenção dos sistemas de saúde e representam uma grande preocupação na atualidade (World Health Organization (WHO), 2008) (Governo do Estado do Rio de Janeiro, 2019).

Ao analisarmos a cultura de segurança do paciente e as implicações das notificações dos EAs na visão profissional e institucional, observa-se o obstáculo em se admitir o "erro" por estarem estes associados à culpa e as penalidades, esquecidos das condutas éticas e legais. Assim, gerenciamento de risco assistencial só é eficaz se a instituição for capaz de olhar suas falhas, sem juízo de valor, fazendo-se compreender que as falhas e os erros não são por culpa isolada de uma pessoa, mas sim de uma problemática sistêmica institucional (Wachter, 2012).

Torna-se essencial reconhecer, minimizar ou eliminar os riscos durante a assistência, através de um eficaz processo de gerenciamento de risco, porém este ainda é um grande desafio para as instituições de saúde. Os danos advindos da prática assistencial comumente estão associados ao erro na confecção/execução ou a violação de um ou mais processos de trabalho. Isso se configura pelos riscos atribuídos às situações, aos produtos ou aos processos e seu potencial em gerar incidentes 
(Agência Nacional de Vigilância Sanitária, (2017), (Borges et al 2020).

$\mathrm{O}$ risco associa-se à probabilidade de ocorrência de um incidente e sua identificação e avaliação torna-se peça fundamental na prevenção de eventos adversos (MS,2014).

Observa-se então, que em virtude de uma determinada probabilidade e magnitude, além de custos, de ocorrência de incidentes ou danos, os riscos podem ser mitigados, isto é, minimizados, ou assumidos e, para isso, há que se ter o gerenciamento desta situação.

Entretanto, pouco sabemos quais são os riscos previsíveis que estão associados aos procedimentos de enfermagem. Há uma escassez de estudos científicos com foco na identificação das punções venosas periféricas realizadas pela enfermagem.

Dentre esses procedimentos elegemos a punção venosa periférica para ser abordado. A escolha se deu por ser a punção venosa periférica o procedimento mais utilizado para se instituir a terapia intravenosa. Mais de $70 \%$ dos pacientes hospitalizados precisam de cateter venoso periférico. (Rickard et al, 2012) Alguns estudos indicam uso de cateter venoso periférico em 86,4\% (New, Webster, Marsh, Hewer, 2014) e 80,6\% (Fernández-Ruiz et al, 2014) dos pacientes.

A maioria dos cateteres são removidos devido a ocorrência de complicações, fim do tratamento ou ausência de uso. (Wallis et al, 2014) Por isso identificar os riscos nos permite desenvolver barreiras para evitar complicações relacionadas à punção venosa periférica, contribuir para prática de cuidados diários seguros e fornecer evidências científicas para tomada de decisão concernente a mitigação dos riscos da terapia intravenosa periférica.

Este protocolo define os passos que serão seguidos na Revisão de Escopo, sobre este tema, e isto permite transparência de todo o processo.

\section{Metodologia}

Trata-se de um protocolo para realização de uma revisão de escopo, estruturada pelo Instituto Joanna Brigs. Este modelo de revisão aprovisiona as evidências que existem sobre o assunto, sem restringir a qualidade metodológica, uma vez que não busca a melhor evidência, mas sim de selecionar e sintetizar o conhecimento existente (Tricco et al, 2018; Colquhoun,2014; Levac, 2010).

O protocolo segue as etapas estabelecidas para uma revisão de escopo fundamentada em orientações do Joanna Briggs Institute (JBI) (Aromataris \& Munn ,2020). Para tal, foi seguida as etapas seguintes:

1) Elaboração da Questão da Pesquisa: A construção da questão de pesquisa foi realizada por meio dos acrônimos PICO (População, Intervenção, Comparação e Resultados), para estudos quantitativos, e PICo (População, Interesse/fenômeno de interesse e Contexto), para estudos qualitativos. Sendo assim, a pergunta de pesquisa que irá nortear a revisão é: "Quais as evidências sobre os riscos e eventos adversos relacionados a punção venosa periférica, realizada por enfermeiros em pacientes hospitalizados?"

2) Construção do protocolo da revisão: Foi desenvolvido este protocolo para detalhar etapas metodológicas que serão seguidas, os métodos e os processos adotados, para reduzir o risco de viés. Sendo realizado o estabelecimento de critérios para inclusão e exclusão de estudos, tipo de participantes, tipo de intervenção/ fenômeno de interesse, tipo de estudos, estratégia de busca, avaliação da qualidade metodológica, extração e análise dos dados.

3) Busca das publicações nas bases de dados eletrônicas: Será realizada a busca dos artigos e seleção das bases eletrônicas de acordo com a viabilidade do acesso. Para seleção dos termos de busca é necessária a identificação dos termos (descritores) relacionados a cada um dos componentes da estratégia PICO ou PICo, com a utilização dos operadores booleanos AND, OR e NOT.

4) Seleção dos artigos: Nesta fase, será realizada a seleção dos estudos de acordo com os critérios previamente definidos. Para maior qualidade dessa fase a checagem dos estudos, será realizada por revisores. 
5) Extração dos dados dos artigos: Ocorrerá a extração dos dados e avaliação dos estudos com o auxílio de um instrumento (Título, nome do autor, local de publicação, fenômeno de interesse, população, metodologia utilizada, resultados e outros). Será avaliado o delineamento de cada pesquisa, sua condução e os resultados encontrados.

6) Síntese e análise dos dados: Nesta fase, os dados serão sintetizados. Os resultados encontrados serão interpretados, avaliados de acordo com o nível de evidência, conforme é recomendado pelo JBI, e discutidos.

Esta revisão de escopo considerará desenhos de estudos experimentais e quase-experimentais, incluindo estudos randomizados e não randomizados. Além disso, estudos observacionais analíticos, incluindo estudos de coorte prospectivos e retrospectivos, estudos de caso-controle e estudos analíticos transversais serão considerados para inclusão. Também serão considerados desenhos de estudos observacionais descritivos, incluindo séries de casos, relatos de casos individuais e estudos transversais descritivos para inclusão.

Além disso, as revisões sistemáticas que atendam aos critérios de inclusão também serão consideradas. Artigos de texto e opinião também poderão ser considerados para inclusão nesta revisão de escopo.

\section{Estratégia de Pesquisa}

A estratégia de busca terá como objetivo localizar estudos publicados e não publicados. Para isso, através da BVS buscou-se conhecer os descritores relacionados ao tema em questão. As palavras do texto contidas nos títulos e resumos de artigos relevantes e os termos de índice usados para descrever os artigos foram usados para desenvolver uma estratégia de busca completa. A estratégia de busca, incluindo todas as palavras-chave e termos de índice identificados, será adaptada para cada banco de dados e / ou fonte de informação incluída. A lista de referência de todas as fontes de evidências incluídas será rastreada para estudos adicionais.

Será realizada pesquisa nas bases de dados: MEDLINE via PubMed, LILACS por meio da Biblioteca Virtual em Saúde (BVS), CINAHL, SCOPUS e WEB OF SCIENCE. Serão desenvolvidas estratégias de busca para cada base ou repositório eletrônico feitos na língua portuguesa (BR), inglesa e espanhola, sem restrição quanto ao ano de publicação. Busca manual em outras bases de dados poderão ser realizadas.

\section{Seleção de Estudo/ Fonte de Evidências}

Após realização da pesquisa, todas as citações identificadas serão agrupadas e carregadas através do gerenciador bibliográfico EndNote, e as duplicatas serão removidas. Após um teste piloto, os títulos e resumos serão selecionados por dois ou mais revisores independentes para avaliação em relação aos critérios de inclusão para a revisão. Fontes potencialmente relevantes serão recuperadas na íntegra e seus detalhes de citação importados para o Sistema JBI para o Gerenciamento Unificado, Avaliação e Revisão de Informações (JBI SUMARI) (JBI, Adelaide, Austrália). O texto completo das citações selecionadas será avaliado em detalhes em relação aos critérios de inclusão por dois ou mais revisores independentes. Os motivos para a exclusão de fontes de evidência no texto completo que não atendam aos critérios de inclusão serão registrados e relatados na revisão de escopo. Quaisquer divergências que surjam entre os revisores em cada etapa do processo de seleção serão resolvidas por meio de discussão ou com um revisor adicional. Os resultados da pesquisa e do processo de inclusão do estudo serão relatados na íntegra na revisão de escopo final e apresentados em um diagrama de fluxo de Itens de Relatório Preferidos para Revisões Sistemáticas e Meta-análises para revisão de escopo (PRISMA-ScR). (Peters et al.,2015) (Peters et al.,2020). 


\section{Extração de Dados}

Os dados serão extraídos de artigos incluídos na revisão de escopo por dois ou mais revisores independentes usando uma ferramenta de extração de dados desenvolvida pelos revisores. Os dados extraídos incluirão detalhes específicos sobre os participantes, conceito, contexto, métodos de estudo e principais conclusões relevantes para a pergunta da revisão.

A ferramenta de extração de dados de rascunho será modificada e revisada conforme necessário durante o processo de extração de dados de cada fonte de evidência incluída. As modificações serão detalhadas na revisão do escopo. Quaisquer divergências que surjam entre os revisores serão resolvidas por meio de discussão e se for necessário, haverá um revisor adicional.

\section{Análise de Dados e Apresentação}

Os dados extraídos serão apresentados em forma de diagrama ou tabular de uma maneira que se alinhe com o objetivo desta revisão de escopo. Tabelas e os mapas irão relatar sobre os riscos e danos da punção venosa periférica. Um resumo narrativo acompanhará os resultados tabulados e mapeados, e descrever como os resultados se relacionam com o objetivo e com a pergunta da revisão.

\section{Considerações Finais}

O objetivo foi alcançado com a criação do protocolo de acordo com o manual da Joanna Briggs. Para trabalhos futuros sugere-se a construção de mais protocolos para subsidiar a produção de revisões de escopo, com metodologias consistentes. Além da realização de mais revisões de escopo referentes aos procedimentos invasivos realizados por enfermeiros.

\section{Referências}

Agência Nacional de Vigilância Sanitária. (2017). Gestão de Riscos e Investigação de Eventos Adversos Relacionados à Assistência à Saúde. Brasil, Brasília: Anvisa.

Aromataris E Munn Z. Chapter 1: JBI Systematic Reviews. In: Aromataris E, Munn Z (Editors). Joanna Briggs Institute Reviewer's Manual. The Joanna Briggs Institute, https://reviewersmanual.joannabriggs.org/

Borges, M. dos S.; Silva, J. L. L. da; Almeida, G. L. de; Santos, L. C. G. dos; Soares, L. M.; Rego, V. T. S. M.; \& Silva, J. V. L. da. (2020) A Enfermagem na prevenção da flebite ocasionada por medicamentos. Research, Society and Development, 9, e317985569, 10.33448/rsd-v9i8.5569.

Colquhoun H. L.; Levac, D., O’Brien, K. K., Straus, S; Tricco, A. C., Perrier , L; Kastner, M.; \& Moher, D. (2014) Scoping reviews: time for clarity in definition, methods, and reporting. 67(12), 1291-1294. 10.1016/j.jclinepi.2014.03.013

Fernández-Ruiz, M., Carretero, A., Díaz, D., Fuentes, C., Gonzáles, J. I., García-Reyne, A., Aguado, J. M., \& López-Medrano, F. 2014. Hospital-wide survey of the adequacy in the number of vascular catheters and catheter lumens. J Hosp Med. [Internet].

Governo do Estado do Rio de Janeiro. (2019). Plano de Fortalecimento das Práticas de Segurança do Paciente na Atenção Primária à Saúde - 2019-2022 /Secretaria de Estado de Saúde; Subsecretaria de Vigilância em Saúde. 67 p.

Kohn, L. T., Corrigan, J. M. \& Donaldson, M. S. (2000). To Err Is Human: Building a Safer Health System. Washington DC: Institute of Medicine.

Levac, D., Colquhoun, H., O'Brien, K. K. (2010)Scoping studies: advancing the methodology. Implementation Science: 5, 69. https://doi.org/10.1186/17485908-5-69

Ministério da Saúde (MS). (2013a). Programa Nacional de Segurança do Paciente (PNSP). Diário Oficial União. Portaria GM/MS N 529, de 01 de abril de 2013.

Ministério da Saúde (MS). (2013b). Portaria GM/MS N 36, de 25 de julho de 2013. Diário Oficial União n 143,25 julho de 2013.

Ministério da Saúde (MS). 2014) Documento de referência para o Programa Nacional de Segurança do Paciente / Ministério da Saúde; Fundação Oswaldo Cruz; Agência Nacional de Vigilância Sanitária. - Brasília: Ministério da Saúde. 40 p.

New, K. A., Webster, J., Marsh, N. M., \& Hewer, B. (2014). Intravascular device use, management documentation and complications: a point prevalence survey. Aust Health Rev. [Internet]. 2014 
Research, Society and Development, v. 10, n. 8, e17510817168, 2021

(CC BY 4.0) | ISSN 2525-3409 | DOI: htttp://dx.doi.org/10.33448/rsd-v10i8.17168

Peters, M. D., Godfrey, C. M., Khalil, H., McInerney, P., Parker, D. \& Soares, C. B. Guidance for conducting systematic scoping reviews. Int J Evid Based Healthc. 13(3):141-6. doi: 10.1097/XEB.0000000000000050. PMID: 26134548.

Peters, M. D. J., Godfrey, C., McInerney, P., Munn, Z., Tricco, A. C., Khalil, H. Chapter 11: Scoping Reviews (2020 version). In: Aromataris E, Munn Z (Editors). JBI Manual for Evidence Synthesis, JBI, 2020. https://synthesismanual.jbi.global. _https://doi.org/10.46658/JBIMES-20-12

Rickard, C. M., Webster, J., Wallis, M. C., Marsh, N., McGrail, M. R., French, V., Foster, L., Gallagher, P., Gowardman, J. R., Zhang, L., McClymont, A., \& Whitby, M. (2012). Routine versus clinically indicated replacement of peripheral intravenous catheters: a randomized controlled equivalence trial. Lancet. [Internet].

Sousa, P. (2014). Segurança do paciente: conhecendo os riscos nas organizações de saúde. EaD/ENSP. 452 p.

Tricco, A. C.; Lillie, E., Zarin, W. al. (25 more authors) (2018) PRISMA Extension for Scoping Reviews (PRISMA-ScR): Checklist and Explanation. Annals of Internal Medicine. pp. 467-73

Wachter, R. M. (2012) Personal accountability in healthcare:searching for the right balance. BMJ Qual Saf; 0:1-5.

Wallis, M. C., McGrail, M., Webster, J., Marsh, N., Gowardman, J., Playford, E. G., \& Rickard, C. M. (2014). Risk factors for peripheral intravenous catheter failure: a multivariate analysis of data from a randomized controlled trial. Infect Control Hosp Epidemiol. [Internet].

World Health Organization (WHO). 2008. World Alliance for Patient Safety. Summary of the evidence on patient safety: implications for research. The Research Priority Setting Working Group of the World Alliance for Patient Safety. Geneva: World Health Organization; 